This item was submitted to Loughborough's Research Repository by the author.

Items in Figshare are protected by copyright, with all rights reserved, unless otherwise indicated.

\title{
Coherence scanning interferometry: measurement and correction of three- dimensional transfer and point-spread characteristics
}

PLEASE CITE THE PUBLISHED VERSION

https://doi.org/10.1364/AO.53.001554

\section{PUBLISHER}

(C) Optical Society of America

\section{VERSION}

AM (Accepted Manuscript)

\section{PUBLISHER STATEMENT}

This work is made available according to the conditions of the Creative Commons Attribution-NonCommercial 4.0 International (CC BY-NC 4.0) licence. Full details of this licence are available at: http://creativecommons.org/licenses/by-nc/4.0/

\section{LICENCE}

CC BY-NC 4.0

\section{REPOSITORY RECORD}

Mandal, Rahul, Jeremy Coupland, Richard K. Leach, and D. Mansfield. 2014. "Coherence Scanning Interferometry: Measurement and Correction of Three-dimensional Transfer and Point-spread Characteristics". figshare. https://hdl.handle.net/2134/34687. 


\title{
Coherence scanning interferometry: measurement and correction of $3 \mathrm{D}$ transfer and point-spread characteristics
}

\author{
Rahul Mandal ${ }^{1}$, Jeremy Coupland ${ }^{1, *}$, Richard Leach ${ }^{2}$ and Daniel Mansfield ${ }^{\mathbf{3}}$ \\ ${ }^{1}$ Wolfson School of Mechanical and Manufacturing Engineering, \\ Loughborough University, Loughborough, LE11 3TU, UK; \\ ${ }^{2}$ Engineering Measurement Division, \\ National Physical Laboratory, TW11 OLW, UK; \\ ${ }^{3}$ Taylor Hobson Ltd, Leicester, LE4 9JQ, UK \\ *Corresponding author: j.m.coupland@lboro.ac.uk
}

Received Month X, XXXX; revised Month X, XXXX; accepted Month X,

XXXX; posted Month X, XXXX (Doc. ID XXXXX); published Month X, XXXX

\begin{abstract}
When applied to the measurement of smooth surfaces, coherence scanning interferometry can be described by a three-dimensional linear filtering operation that is characterized either by the point-spread function in the space domain or equivalently by the transfer function in the spatial frequency domain. For an ideal, aberration-free instrument, these characteristics are defined uniquely by the numerical aperture of the objective lens and the bandwidth of the illumination source. In practice, however, physical imperfections such as lens aberrations, reference focus and source alignment mean that the instrument performance is not ideal. Currently these imperfections often go unnoticed as typically the instrument performance is only verified using rectilinear artefacts, such as step-heights and lateral grids. If an object of varying slope is measured, however, significant errors are often observed as the surface gradient increases. In this paper, a new method of calibration and adjustment using a silica micro-sphere as a calibration artefact is introduced. The silica micro-sphere is used to compute the point-spread and transfer function characteristics of the instrument, and the effect of these characteristics on instrument performance is discussed. Finally, a straightforward method to correct for phase and amplitude imperfections in the transfer function is described using a modified inverse filter.

OCIS Codes: 070.0070, 120.0120, 120.4800, 120.6650, 180.1655, 180.6900
\end{abstract}

\section{Introduction}

With the lateral resolution of a high power microscope and the axial resolution of an interferometer, coherence scanning interferometry (CSI) is an increasingly popular method to measure surface topography [1,2]. Compared to traditional stylus based instrumentation, CSI is non-contacting, easy to use and provides an efficient means to collect the large data sets that are required to estimate areal surface texture parameters [3]. CSI typically exploits broadband, incandescent or LED sources and an interference objective, of Mirau or Michelson type, to record the interference between light reflected from a reference surface and that scattered by the object as it is scanned though focus. Since the source illumination is limited in both temporal and spatial coherence, the interference fringes are observed over a finite scan range and it is relatively straightforward to locate the bright zero order fringe that identifies when path length is balanced in the interferogram. This makes CSI particularly suitable for the measurement of discontinuous surfaces such as those manufactured in the microelectronics industry.

Despite these significant advantages, it has been noted by several authors that surface parameters reported by CSI can vary considerably from those obtained by contacting metrology; particularly when there are appreciable changes in surface gradient [4, $5]$. An example of this type of error, that was observed in the measurement of a sinusoidal grating of $8 \mu \mathrm{m}$ pitch and $466 \mathrm{~nm}$ peak-to-peak amplitude, is shown in figure 1. It is clear that the measured output (solid line) approximately follows the ideal profile (dotted line) in regions of low gradient (i.e. the peaks and troughs).

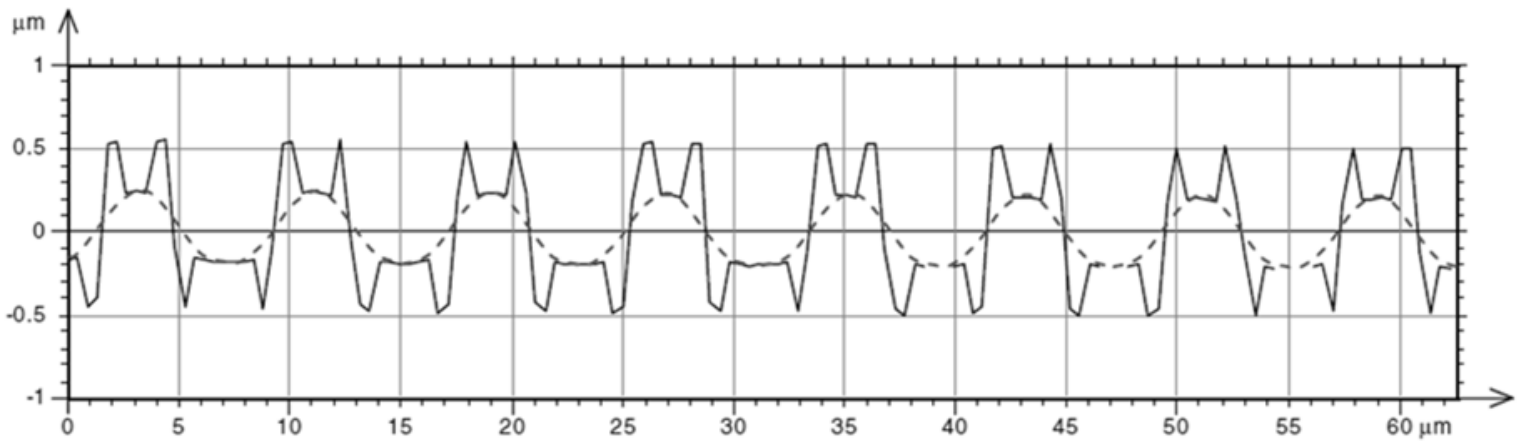

Figure 1: Measurement of sinusoidal profile using CSI 
As the gradient increases, fringe order (or $2 \pi$ ) errors become more prevalent resulting in spurious peaks in the measured peak-to-peak amplitude. Interestingly, detailed inspection shows that the measurement systematically underestimates the curvature of the troughs and overestimates that of the peaks in the sinusoidal profile. Gradient-related errors such as these have brought into question the use of CSI as a traceable measurement tool and for this reason contacting metrology is often used for primary standard instruments (see for example, [6]).

It has been noted elsewhere that gradient-related errors are closely correlated to the frequency response of the system [7]. The frequency response or transfer function has been defined in several ways, however, and it is useful to discuss these briefly here. The instrument transfer function (ITF) characterizes the $2 \mathrm{D}$ response of the whole system to the function, $s\left(r_{x}, r_{y}\right)$, that describes the surface topography [8]. It is important to note that the ITF is only relevant to small departures from a planar surface $(s<\lambda / 8)$ since the system as a whole is highly non-linear otherwise [9]. The ITF can be used to determine the instrument spatial frequency cut-off and is closely associated with errors observed at step discontinuities [7]; but it does not explain why gradient-related errors occur. The optical transfer function (OTF) is a characteristic that describes the frequency response of the imaging system. The OTF is also defined in $2 \mathrm{D}$ and relates the intensity of the field in the object plane to that observed at the image plane. Equivalently the OTF can be defined in terms of the complex amplitude of the fields in which case it is more often called the coherent transfer function (CTF) [10]. The 2D CTF explains why the system is restricted in terms of gradient as the spatial frequency content of the amplitude field exceeds the cut-off frequency; but it is not clear how the field in a given plane is related to the surface topography.

In order to understand the errors associated with CSI and other optical instruments it is useful to determine their 3D transfer characteristics $[11,12]$. In a recent work the process of scattering from the smooth interface between homogenous materials was considered, and it was shown that, with appropriate assumptions, CSI can be described by a 3D filtering operation [13]. In this work, the surface is characterized by an infinitely thin membrane called the "foil model" of the surface. The corresponding filtering operation is characterized in the frequency domain by the $3 \mathrm{D}$ transfer function which, for an ideal system, is determined uniquely by the numerical aperture (NA) of the objective lens and the bandwidth of the illumination source. The foil model clearly shows the link between the transfer function and gradientdependent measurement errors. Furthermore, it is straightforward to introduce the effect of aberration on the 3D transfer function [14].

It is noted that although there is a clear relationship between measurement errors and surface gradient, errors often go unnoticed in practice since it is usual to calibrate CSI instrumentation using rectilinear artefacts such as step heights and lateral grids of known geometry $[15,16]$. While this type of artefact is appropriate to stepped surfaces such as etched micro- electronic components, it is insufficient to estimate uncertainty in the measurement of sloped surfaces.

In this paper, calculation of the $3 \mathrm{D}$ transfer characteristics from measurements of a spherical calibration artefact is discussed and, in certain cases, phase and amplitude errors can be corrected. This process forms the basis of a calibration and adjustment protocol for CSI instrumentation.

The paper is organized as follows: In section 2 , the linear theory of 3D imaging is briefly introduced and the 3D transfer function (TF) and point spread function (PSF) are defined with reference to the foil model of the object surface. The measurement of these characteristics using a spherical artefact is then explained in Section 3. Section 4 describes how a modified inverse filter can be designed to compensate the systematic errors present in the system and the application of this adjustment protocol is presented Section 5 .

\section{Theory}

Mathematically, the output, $O(\mathbf{r})$, of a CSI instrument can be written as a $3 \mathrm{D}$ linear filtering operation that is characterized in the space domain by the convolution [13],

$$
O(\mathbf{r})=\int H\left(\mathbf{r}-\mathbf{r}^{\prime}\right) \Delta\left(\mathbf{r}^{\prime}\right) d^{3} r^{\prime}
$$

where $H(\mathbf{r})$ is the point-spread function (PSF). Equivalently, in the frequency domain ( $\mathrm{k}$-space) the filtering operation is characterized by the product,

$$
\tilde{O}(\mathbf{k})=\tilde{\Delta}(\mathbf{k}) \widetilde{H}(\mathbf{k})
$$

where tilde denotes Fourier transformation such that the transfer function (TF) is $\widetilde{H}(\mathbf{k})=\int H(\mathbf{r}) e^{-2 \pi \mathbf{k} \cdot \mathbf{r}} d^{3} r$. In these expressions $\Delta(\mathbf{r})$ and $\tilde{\Delta}(\mathbf{k})$ are functions that define the $3 \mathrm{D}$ object distribution in the frequency and space domains respectively. Provided the surface is smooth, such that at any point the radius of curvature is greater than the wavelength, the interface between of two homogeneous media can be represented as a thin foil-like object such that,

$$
\Delta(\mathbf{r})=4 \pi j R W\left(r_{x}, r_{y}\right) \delta\left(r_{z}-s\left(r_{x}, r_{y}\right)\right)
$$

where, $R$ is the reflection coefficient, $W\left(r_{x}, r_{y}\right)$ is a window function that defines the illuminated area and $s\left(r_{x}, r_{y}\right)$ is the $2 \mathrm{D}$ function that defines the height of the surface of interest. To illustrate the foil model, a micro-sphere and the foil model of a region from its upper surface is shown in figure 2 .

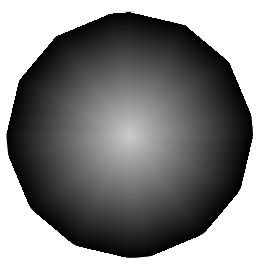

(a)

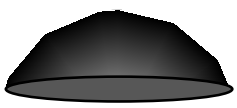

(b)
Figure 2: a) Micro-sphere and b) foil model 
For a perfect conductor the reflection coefficient is independent of incidence angle and equal to unity. In general, however, the reflection coefficient is complex, polarisation-dependent and varies slightly with incidence angle. Nevertheless, increased reflection of one polarisation state is approximately compensated by a decrease in the other and, over the range of NA of interest to CSI $(\mathrm{NA}<0.7)$, to a good approximation the reflection coefficient can be replaced by its value at normal incidence, $R=(1-\mathrm{n}) /(1+\mathrm{n})$ such that,

$$
\Delta(\mathbf{r}) \approx 4 \pi j\left(\frac{1-\mathrm{n}}{1+\mathrm{n}}\right) W\left(r_{x}, r_{y}\right) \delta\left(r_{z}-s\left(r_{x}, r_{y}\right)\right) .
$$

Equation 4. defines the foil model of the surface [13]. With this definition it can be shown that, for a CSI system using a source of spectral density, $S\left(k_{0}\right)$, (represented as a function of wavenumber, $k_{0}=1 / \lambda$ ), the $3 \mathrm{D}$ transfer function, $\widetilde{H}(\mathbf{k})$, is given by

$\widetilde{H}(\mathbf{k})=\left(\frac{|\mathbf{k}|^{2}}{2 \mathbf{k} . \widehat{\mathbf{o}}}\right) \iint \tilde{G}_{N A}\left(\mathbf{k}_{\mathbf{r}}, k_{0}\right) \tilde{G}_{N A}(\mathbf{k}-$

$\left.\mathbf{k}_{\mathbf{r}}, k_{0}\right) d^{3} k_{r} S\left(k_{0}\right) d k_{0}$

where $\tilde{G}_{N A}\left(\mathbf{k}_{\mathbf{r}}, k_{0}\right)$ is the Green's function of the imaging system (equivalent to the $3 \mathrm{D} \mathrm{CTF}$ ) expressed in the frequency domain and is given by,

$\tilde{G}_{N A}\left(\mathbf{k}, k_{0}\right)=\frac{j}{4 \pi k_{0}} \delta\left(|\mathbf{k}|-k_{0}\right) \operatorname{step}\left(\frac{\mathbf{k} . \widehat{o}}{k_{0}}-\sqrt{1-N A^{2}}\right)$

where $\widehat{\mathbf{o}}$ is a unit vector in the direction of the optical axis and $\delta(x)$ and $\operatorname{step}(x)$ represent a Dirac delta function and a Heaviside step function respectively.

Equations 2, 4, 5 and 6 define the output of an ideal CSI in terms of its NA and source spectrum. Figure 3 illustrates the TF and PSF of an ideal instrument having NA $=0.55$, a mean wavelength of $\lambda=600 \mathrm{~nm}$ and a Gaussian spectral density, $S\left(k_{0}\right)$, with a bandwidth of $120 \mathrm{~nm}$ (FWHM at $1 / e^{2}$ ).

According to equations 5 and 6 , the lateral extent ( $y$ direction) of the TF (figure 3a) is determined by the system NA while the axial extent ( $z$ direction) is dictated by both the NA and the source bandwidth. The corresponding PSF shown in figure $3 \mathrm{~b})$ is essentially a packet of fringes and (according to the Fourier transform relationship) its dimensions are inversely proportional to those of the TF. In this case the lateral dimension is approximately $0.5 \mu \mathrm{m}$ and its axial extent is approximately $1.5 \mu \mathrm{m}$ (both FWHM at $\left.1 / \mathrm{e}^{2}\right)$. It is noted that the dimensions of the PSF provide a direct measure of the $3 \mathrm{D}$ resolution of a CSI system that determines the surface position by way of the fringe envelope. If phase estimation is used, however, a comparable measure of resolution is the fringe spacing or mean effective wavelength [17], which is approximately $300 \mathrm{~nm}$ in this case.

As a final point, it should be noted that there is a maximum surface gradient that can be measured using a CSI instrument. This is due to the convolutional form of equation 1 , which means that the output of CSI at a given point can be viewed as the PSF integrated over the surface defined by an appropriately positioned foil. If the foil surface is normal to the optical axis it will pass through a single fringe and the absolute value of the surface integral will be a maximum. If, however, the surface is tilted the foil will pass through several fringes and the surface integral will be substantially reduced. Equivalently, considering the Fourier transform of a tilted surface in $\mathrm{k}^{-}$-space, it is straightforward to show that the maximum surface gradient, $\theta_{\max }$, is given by

$$
\theta_{\max }=\sin ^{-1}\left(k_{y} / k_{z}\right)_{\max }
$$

where $\left(k_{y} / k_{z}\right)_{\max }$ takes its maximum value for any non-zero valued point within the TF and, due to the Fourier transform relationship, is directly proportional to the ratio of the minimum fringe spacing to the lateral extent of the PSF.

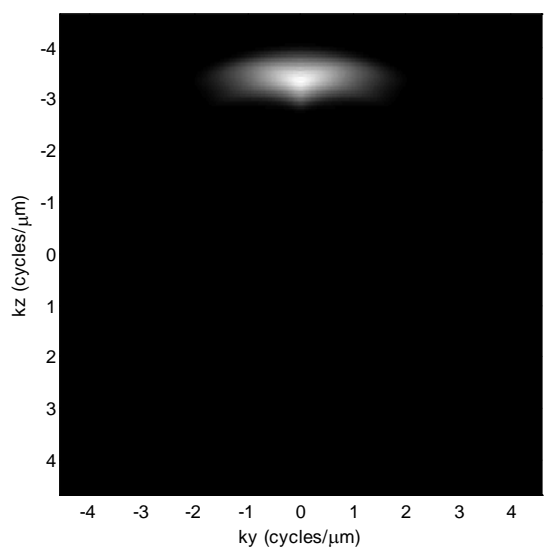

(a)

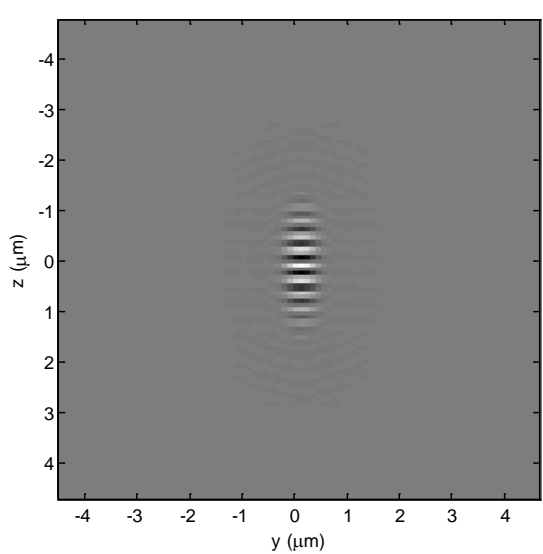

(b)

Figure 3: Sections through a) TF (absolute value) and b) PSF (real part)

From the discussions above it is clear that the 3D PSF/TF characteristics are directly related to the performance of CSI instruments. In practice, however, the PSF/TF can depart significantly from the ideal characteristics due to the effect of aberrations. For example it is often possible to defocus the reference arm of a high power objective and this can significantly change the lateral resolution and maximum measurable surface gradient of the instrument. In a previous publication, the effect of axial (shift invariant) aberrations (defocus, spherical and axial chromatic aberration) on CSI have been modelled and errors similar to those reported in figure 1 have been observed [14]. It can be concluded from this work that in order to properly characterize performance it is essential to calibrate CSI instruments using artefacts with sloped surfaces. In the following section a method 
to measure the TF/PSF characteristic using a spherical artefact is discussed.

\section{Measurement of PSF/TF characteristics}

By definition, the PSF is the response of the system to an ideal point-like object. Although a particle that is significantly smaller than a wavelength might be considered to be a suitable candidate for a calibration artefact, it would be difficult to implement in practice, would have poor scattering efficiency and moreover, invalidates the fundamental assumptions of the foil model. As mentioned previously, the foil model rests on the assumption that the object is smooth such that the radius of curvature exceeds the wavelength. In this case, if the form of the calibration artefact, $\tilde{\Delta}_{c a l}(\mathbf{k})$, is known, the instrument TF, $\widetilde{H}_{\text {inst }}(\mathbf{k})$, is given by

$$
\widetilde{H}_{i n s t}(\mathbf{k})=\frac{\tilde{O}_{c a l}(\mathbf{k})}{\tilde{\Delta}_{c a l}(\mathbf{k})}
$$

where $\tilde{O}_{c a l}(\mathbf{k})$ is the Fourier transform of the interferogram that is output by the instrument while measuring the calibration artefact. It is clear from this expression that the calibration artefact must be chosen such that $\tilde{\Delta}_{c a l}(\mathbf{k})$ adequately covers the frequency domain, such that $\left|\tilde{\Delta}_{c a l}(\mathbf{k})\right|>0$ for all $\left|\tilde{O}_{c a l}(\mathbf{k})\right|>0$.

In practice, a spherical surface that fits within the instrument's field of view is ideally suited to this task. To the authors' knowledge, however, a spherical calibration artefact with suitable form deviation (subnanometre) is not commercially available. In previous work a small mercury droplet deposited on glass was reported for this task since surface tension demands a spherical form to the required tolerance [18]. For routine calibration, a more stable transferable artefact is required and for this reason a calibration and adjustment protocol for CSI instrumentation based on measurements of silica micro-spheres was investigated.

The spheres used in this work were NIST standard silica micro-spheres of diameter $53 \mu \mathrm{m} \pm 1 \mu \mathrm{m}$, purchased from Corpuscular Inc., Cold Spring, NY 10516. Although neither the diameter nor the form of these particles is known to the required tolerance, the manufacturing process ensures that the shape is spherical. In principle, the sphericity can be determined using a statistical argument by measuring the form at different orientations using, for example, an un-calibrated CSI instrument [19]. For the purpose of illustrating the calibration process, however, in the following it is assumed that the calibration artefact is an exact silica sphere that is in direct contact with a planar silica substrate. Note that there is work at the National Physical Laboratory to develop a primary instrument to measure the form of micro-spheres with an uncertainty of approximately $1 \mathrm{~nm}$.

The calibration procedure was as follows:

i) The scales of the CSI instrument were calibrated using a $1.844 \mu \mathrm{m} \pm 0.011 \mu \mathrm{m}$ step height and $9.996 \mu \mathrm{m} \pm 0.019 \mu \mathrm{m}$ pitch lateral grid (from VLSI) to determine the $x y z$ amplification coefficients.

ii) With the micro-sphere centred approximately within the field of view, an interferogram was recorded. iii) The interferogram was processed to remove electronic noise by a) introducing a Hanning window, b) calculating the amplitude spectrum and c) setting all frequency components outside of the pass-band defined by the theoretical TF (equation 5) to zero. A $y z$ section through the inteferrogram and its spectrum before filtering are shown in figure $4 \mathrm{a})$ and $4 \mathrm{~b}$ ), while the images of the band-pass filter and the fringe after filtering are shown in figure 5a) and 5b) respectively.

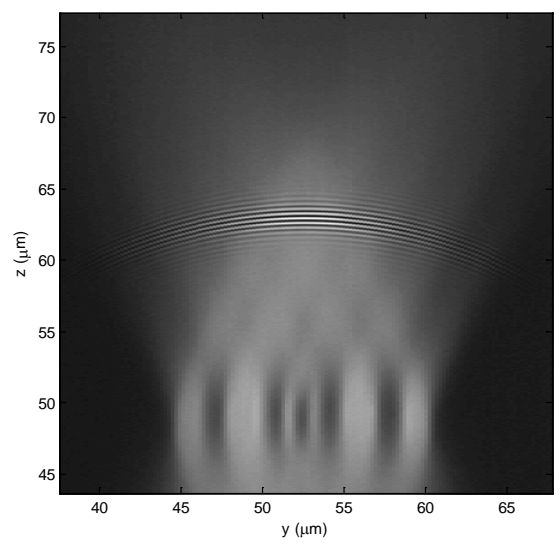

(a)

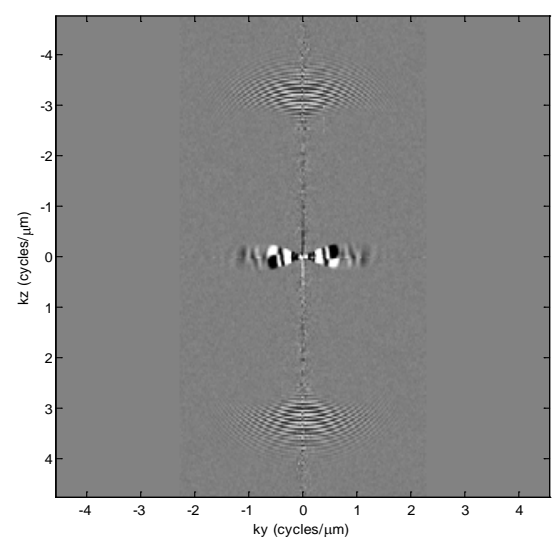

(b)

Figure 4: Sections through a) interferogram and b) its spectrum (real part)

iv) The diameter of a silica micro-sphere was determined using the CSI instrument by measuring the distance between the top of the sphere and the plane of best fit to the substrate surface. By this method the mean diameter of the micro-sphere used for this work was found to be $53.626 \mu \mathrm{m} \pm 0.005 \mu \mathrm{m}$. It is noted that this measurement rests on the assumption that the sphere is in direct contact with the substrate and the difference between the radius of curvature at the top surface and the substrate does not significantly affect the measurement.

v) Using the diameter obtained from step iv), the foil model of the surface was generated. It is noted here that the $1 \mathrm{D}$ delta function that defines the foil model of the surface (equation 4) has infinite bandwidth and to avoid aliasing in the numerical analysis a $1 \mathrm{D}$ 
Gaussian function was used to define the profile of the surface in the $z$ direction. The position of the modified foil surface was approximately aligned with the central fringe in the interferogram. The modified foil surface is shown in figure 6 .

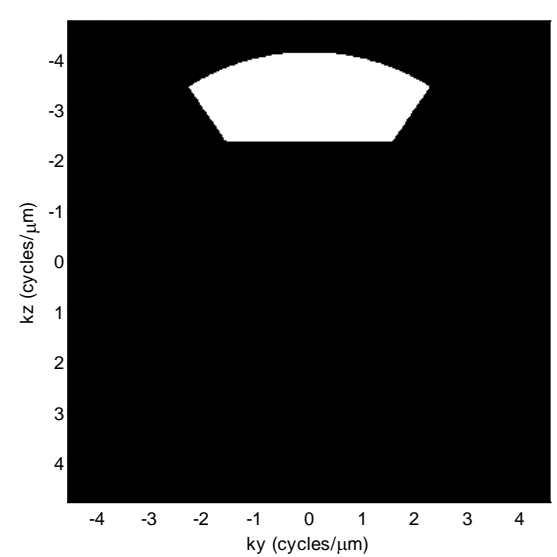

(a)

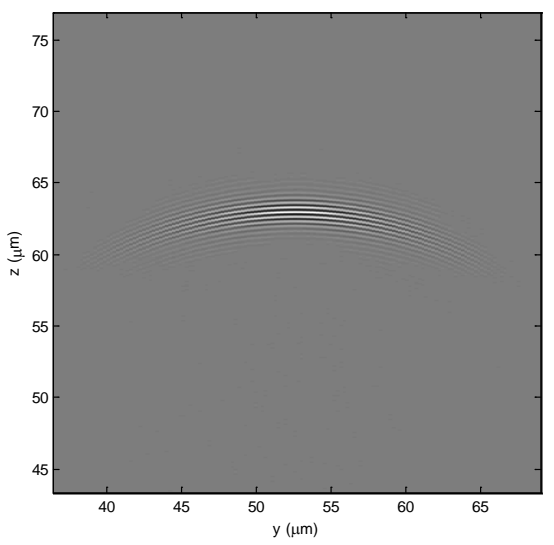

(b)

Figure 5: Sections through the a) filter and b) fringe pattern after filtering (real part)

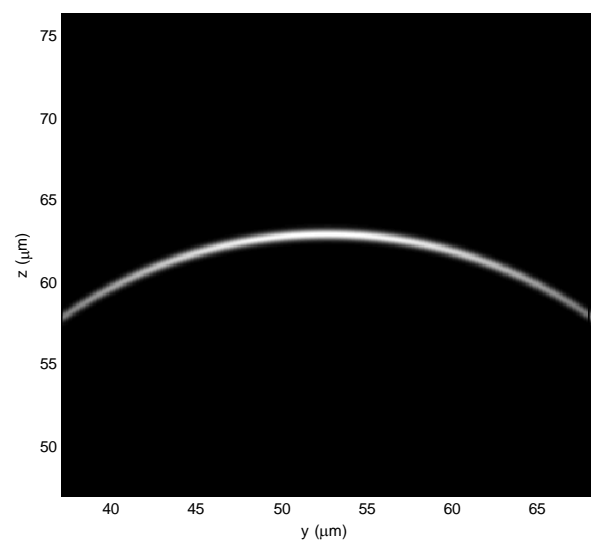

Figure 6: Sections through the foil model of the surface

The transfer function was then calculated by dividing the Fourier transform of the interferogram by that of the modified foil, as defined by equation 8 . The result is shown in figure 7. vii) Finally, the PSF was calculated by inverse Fourier transformation of the $\mathrm{TF}$ shown in figure 7 and is illustrated in figure 8 .

Comparison of figure 7 with the ideal $\mathrm{TF}$ shown in figure 3a) reveals that the measured TF does not extend to the same extent laterally and consequently both the lateral resolution and maximum gradient that can be measured by the system, is less than ideal. Comparing the PSF shown in figure 7 with that in figure $3 \mathrm{~b}$ ) it can be seen that the lateral extent of the instrument PSF is increased resulting in lower resolution. The increased axial extent makes identification of the zero order fringe more difficult and increases the likelihood of fringe order errors.

Although the extent of the TF can be used to provide an estimate of resolution and measurable gradient limitations, its phase has an even greater importance if it is used to determine the position of the surface. According to equation 5, the $\mathrm{TF}$ of an ideal system is real valued. Figure 7b) shows, however, that although the real value dominates, the imaginary value is nonzero and consequently there is a small but significant phase variation across the measured TF. It is possible, however, to use inverse filtering techniques to correct phase errors and boost the amplitude response of CSI instrumentation as described in the following section.

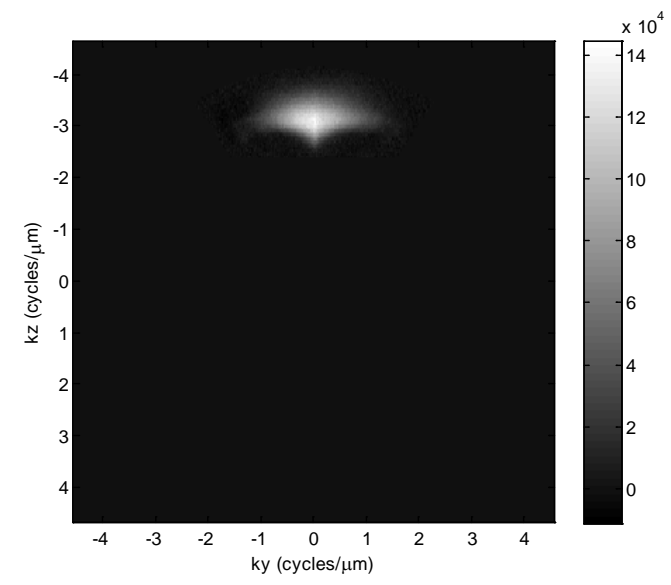

(a)

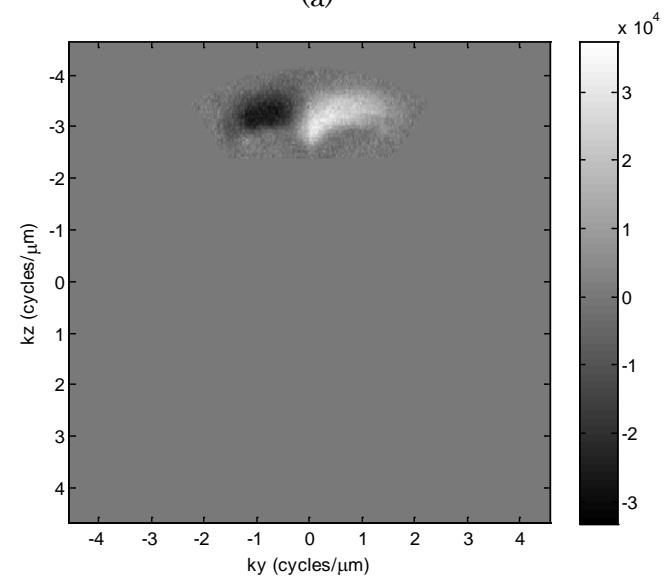

(b)

Figure 7: Sections through the measured TF a) real part and b) imaginary part 


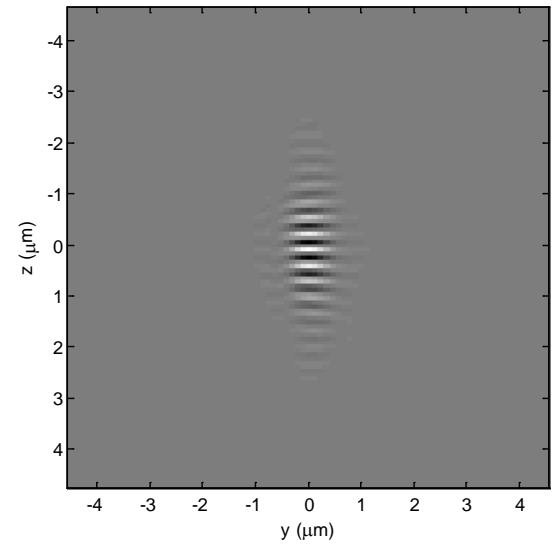

Figure 8: Sections through the measured PSF (real part)

\section{Correction of PSF/TF using inverse filtering}

An inverse filter attempts to correct for phase and amplitude errors in a system response and is commonly used in digital image processing for image de-blurring [20]. If the measured transfer function is $\widetilde{H}^{\text {meas }}(\mathbf{k})=A(\mathbf{k}) \exp (j \varphi(\mathbf{k}))$, then an inverse filter has transfer function, $\widetilde{H}^{i n v}(\mathbf{k})$, that is given by,

$$
\widetilde{H}^{i n v}(\mathbf{k})=\frac{1}{A(\mathbf{k})} \exp (-j \varphi(\mathbf{k}))
$$

An inverse filter compensates for the phase variation and boosts the amplitude with a gain, $1 / A(\mathbf{k})$, in an attempt to flatten the frequency response. For CSI, this has the effect of reducing the lateral dimensions of the PSF and consequently increasing the resolution. In practice, however, the gain should not be allowed to take any value as a significant boost results in a corresponding decrease in signal-to-noise ratio (SNR). For this reason, a modified inverse filter was designed to limit the gain to ten times before dropping to unity gain. The transfer function of the modified filter, $\widetilde{H}^{\text {mod }}(\mathbf{k})$, was defined such that,

$$
\widetilde{H}^{\bmod }(\mathbf{k})=\frac{\exp (-j \varphi(\mathbf{k}))}{W(\mathbf{k})}
$$

where $W(\mathbf{k})$ is a weighting function given by,

$W(\mathbf{k})=\left(A_{n}(\mathbf{k})+0.027\right)\left(1+\exp \left(-A_{n}(\mathbf{k}) \times 58+3.9\right) / 1.5\right.$ and $A_{n}(\mathbf{k})=A(\mathbf{k}) / \max [A(\mathbf{k})] \quad$ is the normalised amplitude. The modified inverse filter is a combination of sigmoid curve and a general inverse filter. The coefficients of the weighting function are calculated to satisfy the specification described before. A plot of the gain of the modified inverse filter as a function of normalized amplitude is shown in figure 9 .

The modified inverse filter is applied in the frequency domain and is multiplied by the Fourier transform of the interferogram. Figure 10 shows a) a $y z$ section through a modified spectrum and b) the resulting modified interferogram of a silica microsphere.

Comparing figure 5b) with figure 10b) it can be seen that the amplitude of the fringes is greater at larger tilt angles and the envelope is also reduced. This is mainly due to the gain of the modified inverse filter boosting the frequencies at the edge of the pass band and, to a limited extent, by the phase correcting properties of the filter. The following section discusses these effects further.

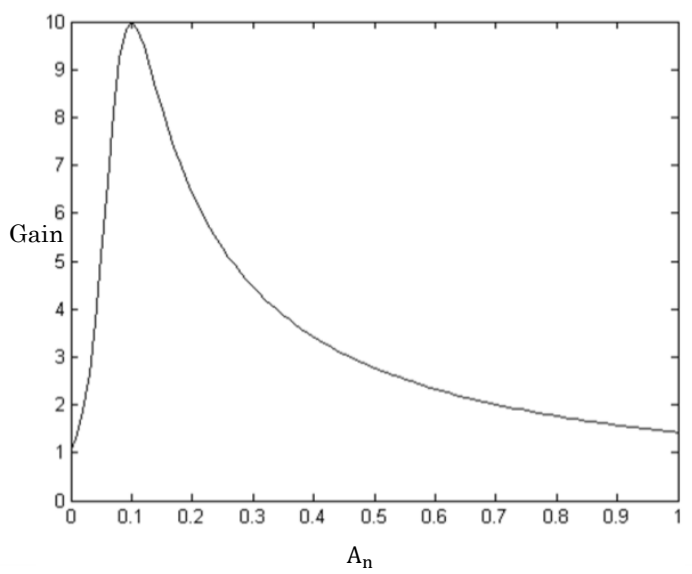

Figure 9: Gain of the weighting function

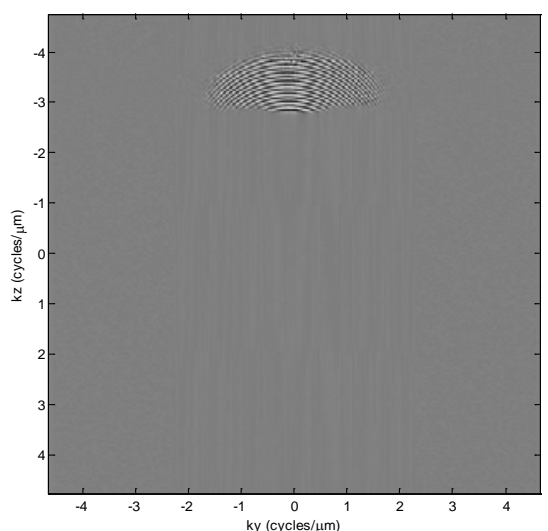

(a)

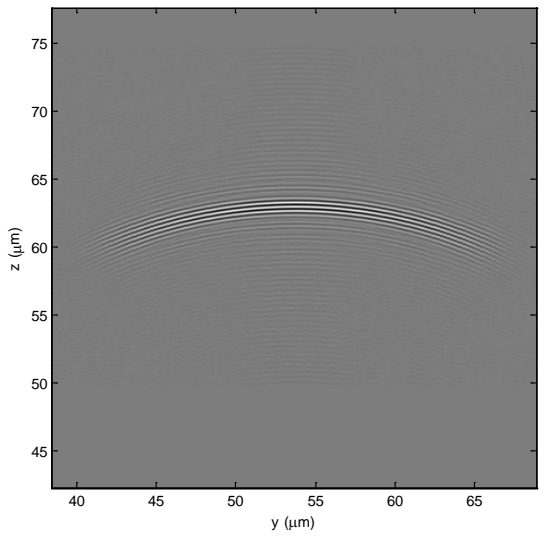

(b)

Figure 10: Sections through the a) corrected spectrum (real part) and b) corresponding fringes (real part)

\section{Application of the modified inverse filter}

Once the transfer characteristics are measured the inverse filter can be designed and it is straightforward to apply the filter to subsequent interferograms recorded by the CSI instrument. This, in essence, is a process of calibration and adjustment. To illustrate the effect of this process, the modified inverse filter was applied to the same micro-sphere that was used to measure the transfer characteristics. The surface height was estimated using the frequency domain 
analysis (FDA) method [21] and this was subtracted from the ideal form of the sphere to give the error surface.

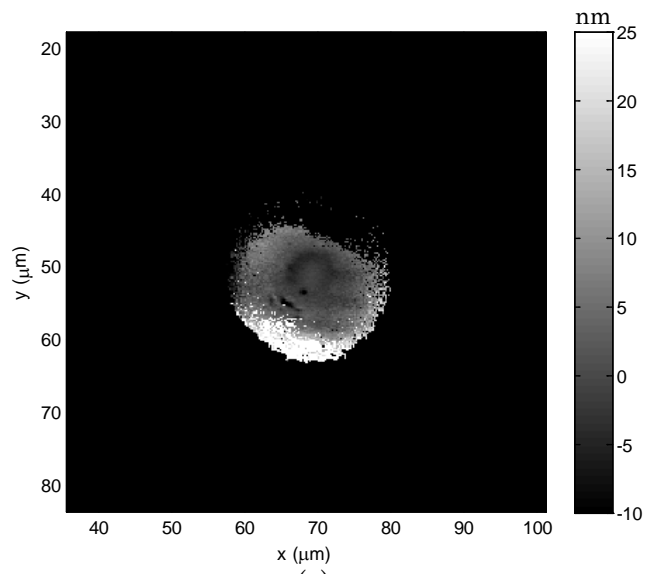

(a)

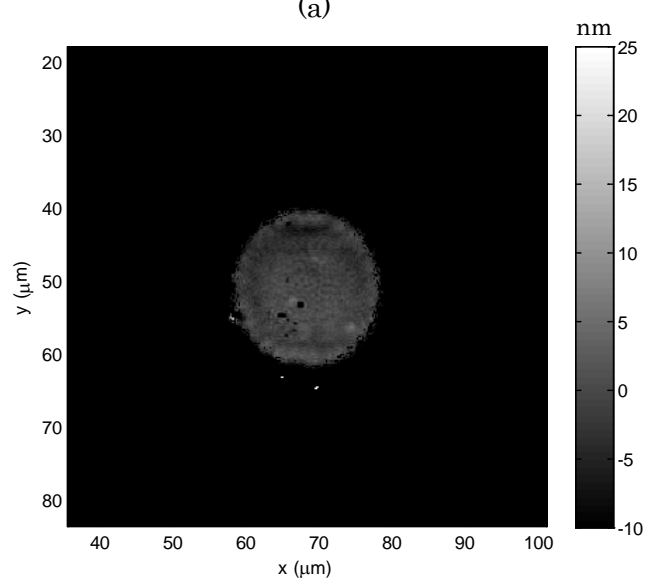

(b)

Figure 11: Error surface for the surface extracted from (a) instrument measurements (b) corrected fringes

In figure 11 the surface obtained from the interferogram, filtered using the modified inverse filter is compared with that obtained from the instrument. It can be seen that before filtering, a height variation in the range of $-10 \mathrm{~nm}$ to $25 \mathrm{~nm}$ and fringe order or $2 \pi$ errors at the top and bottom (outside of the scale) is observed in the error surface. Using the modified inverse filter no fringe order errors are apparent and the form error is reduced to approximately $2 \mathrm{~nm}$ ( $\mathrm{rms}$ ).

It is perhaps not surprising that the modified inverse filter was able to correct errors in the measurement of the sphere which was used to construct it. A more challenging task is to correct interferograms of known objects placed at different places within the field of view thereby establishing that the calibration and adjustment process is shift invariant. To examine this property, interferograms of the test micro-sphere were obtained at the approximate locations shown in figure 12.

The previous result shown in figure 11 is that obtained at the centre of the field - position A. In the same manner Figures 13 and 14 show comparison of the error surfaces found with and without the use of the modified inverse filter for spheres located at the edge of the field. It can be seen that a similar reduction in the measurement error is possible.

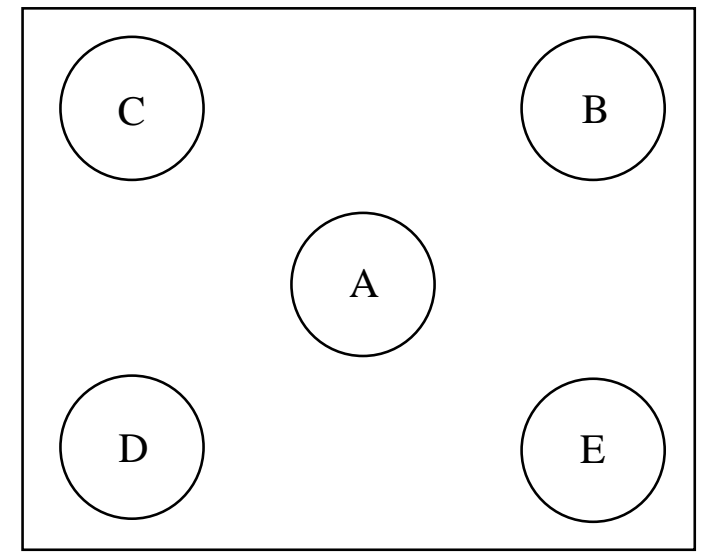

Figure 12: Position of the droplets in total field of view

\section{Conclusions}

It has recently been shown that the interferogram that is output by a CSI instrument can be described by a linear filtering operation that is characterized by the $\mathrm{PSF}$ in the space domain or equivalently the TF in the frequency domain. The linear filter is applied to a foillike membrane placed at the sample interface which is called here the foil model of the surface. The 3D $\mathrm{PSF} / \mathrm{TF}$ characteristics are directly related to the resolution of the instrument and also define the maximum surface gradient that the instrument can measure.

In this paper a method to measure and correct the 3D TF/PSF characteristics of a CSI instrument was presented. The proposed method goes beyond the standard rectilinear calibration methods that use step height and lateral grid artifacts to determine the lateral and axial magnifications of the instrument respectively. In this case a calibration artifact with a continuously varying surface gradient - a silica microsphere - is used to determine the PSF/TF characteristics. A modified inverse filter has been demonstrated as a means to correct non-ideal PSF/TF characteristics. This filter is straightforward to apply and significantly improves the fidelity of the fringes observed in the interferogram. Moreover, phase changes which are directly related to errors in surface height measurement are compensated by this calibration and adjustment procedure. Although further work is necessary to determine the statistical uncertainty of the resulting measurements, these preliminary findings suggest an uncertainty of $1 \mathrm{~nm}$ to $2 \mathrm{~nm}$ is possible in surface measurements made using typical CSI instrumentation if the form of the calibration artefact is known.

\section{Acknowledgments}

The authors gratefully acknowledge support and funding from Taylor-Hobson Ltd. and NPL (under the UK National Measurement System Engineering \& Flow Metrology Programme) for this work. We also acknowledge useful discussions with Kanik Palodhi, Andrew Henning and Claudiu Giusca. 


\section{REFERENCES}

1. B. S. Lee, and T. C. Strand, "Profilometry with a coherence scanning microscope", Appl. Opt., 29, 3784-3788 (1990)

2. L. Deck, and P. De Groot, "High-speed noncontact profiler based on scanning white-light interferometry”, Appl. Opt., 33, 7334-7338 (1994)

3. R. K. Leach, "Characterisation of Areal Surface Texture" (Springer-Verlag, 2013).

4. F. Gao, R. K. Leach, J. Petzing, and J. M. Coupland, "Surface measurement errors using commercial scanning white light interferometers", Meas. Sci. Technol., 19, 015303 (2008)

5. T. V. Vorburger, H.G. Rhee, T. B. Renegar, J.-F. Song, and A. Zheng, "Comparison of optical and stylus methods for measurement of surface texture," Int. J. Adv. Manuf. Technol. 33, 110-118 (2007).

6. R. K. Leach, C. L. Giusca, K, Naoi, "Development and characterisation of a new instrument for traceable measurement of areal surface texture", Meas. Sci. Technol., 20, 125103 (2009)

7. W. Xie, P. Lehmann, and J. Niehues, "Lateral resolution and transfer characteristics of vertical scanning white-light interferometers," Appl. Opt. 51, 1795-1803 (2012).

8. P. De Groot, X. C. Lega, "Interpreting interferometric height measurements using the instrument transfer function", Proceedings of Fringe 2005 Ed. W. Osten, 30-37 (Springer Berlin Heidelberg 2006)

9. G. Häusler and S. Ettl, "Limitations of optical 3Dsensors," in Optical Measurement of Surface Topography, R. Leach, 23-48. Ed (SpringerVerlag, 2011),

10. J. D. Gaskill, "Linear Systems, Fourier Transforms, and Optics", Wiley, New York, 456520 (1978).

11. J. M. Coupland, J. Lobera, "Holography, tomography and 3D microscopy as linear filtering operations", Meas. Sci. Technol., 19(7), 07010 (2008)

12. M. R. Foreman, Giusca C. L., Coupland. J. M., Török P., Leach R. K., "Determination of the transfer function for optical surface topography measuring systems - a review", Meas. Sci. Technol., 24, 052001 (2013)

13. J. M. Coupland, R. Mandal, K. Palodhi, R. K. Leach, "Coherence scanning interferometry: linear theory of surface measurement", Appl. Opt., 52, 3662-3670 (2013)

14. K. Palodhi, J. M. Coupland, and R. K. Leach, "A Linear model of fringe generation and analysis in coherence scanning interferometry", presenterd at the ASPE Summer Topical Meeting on Precision Interferometric Metrology June 23-25, 2010 Asheville, North Carolina.

15. C. L. Giusca, R. K. Leach, Helery F., "Calibration of the scales of surface topography measuring instruments: Part 2. Amplification, linearity and squareness", Meas. Sci. Technol., 23, 065005 (2012)

16. A. Henning, C. Giusca, A. Forbes, I. Smith, R. K. Leach, J. M. Coupland, R. Mandal, , "Correction for lateral distortion in coherence scanning interferometry", CIRP Annals Manufacturing Technology 62 , 547-550 (2013)

17. J. W. Gates, "Fringe spacing in interference microscopes, J. Sci. Instrum. 33, 507, 1956.

18. R. Mandal, K. Palodhi, J. M. Coupland, R. K. Leach, D. Mansfield, "Application of linear systems theory to characterize coherence scanning interferometry", Proc. SPIE, 8430, 84300T (2012)

19. Y. Zhou, Y. Ghim, A. Fard, and A. Davies, "Application of the random ball test for calibrating slope-dependent errors in profilometry measurements," Appl. Opt. 52, 5925-5931 (2013).

20. W. K. Pratt, "Digital Image Processing": Second Edition, John Wiley \& Sons, Inc.. 351-354 (2001).

21. P. De Groot, and L. Deck, "Surface profiling by analysis of white-light interferograms in the spatial frequency domain”, J. Mod. Opt., 42, 389401 (1995) 

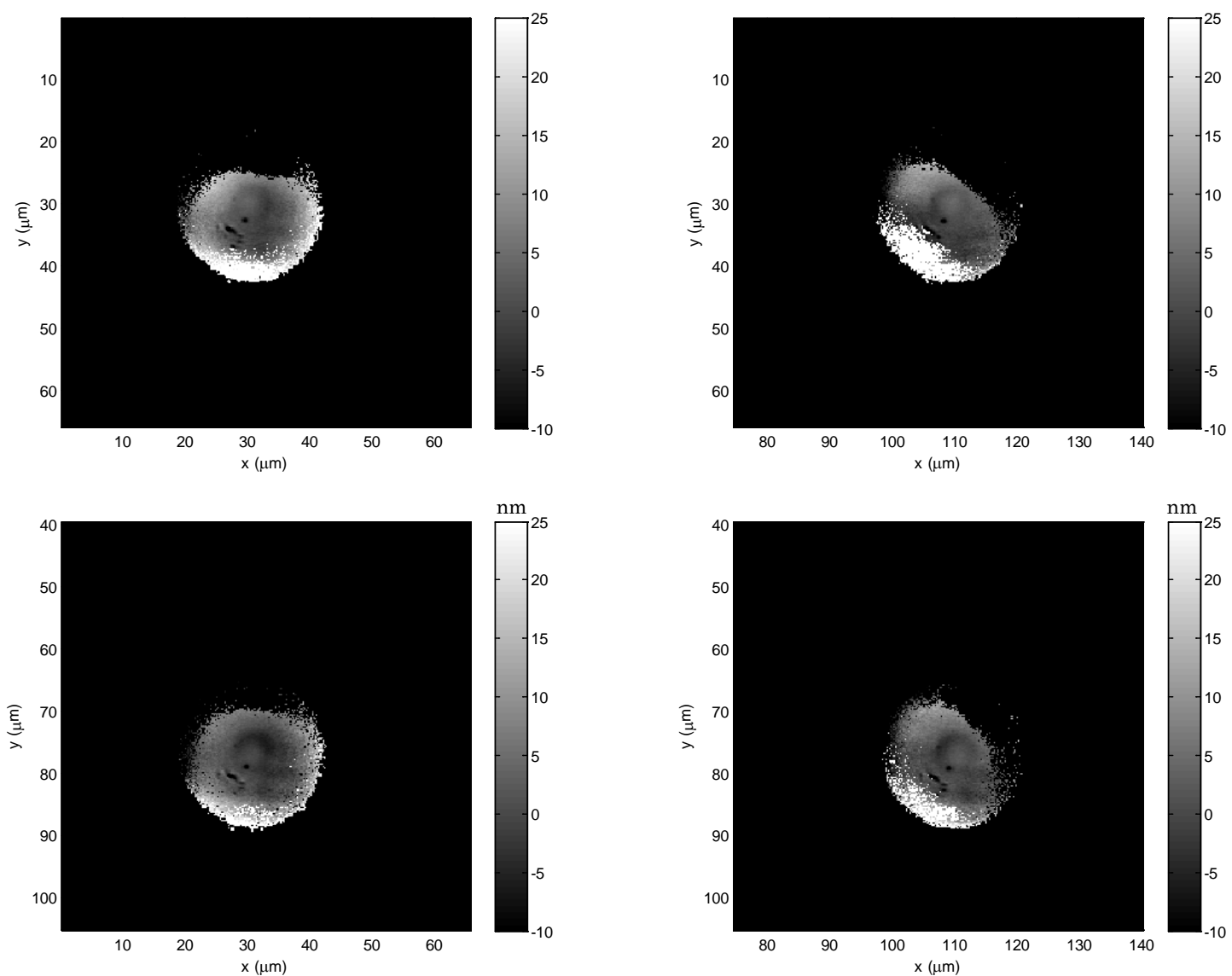

Figure 13: Error surfaces extracted from instrument measurements at positions CBDE (clockwise from top left) 

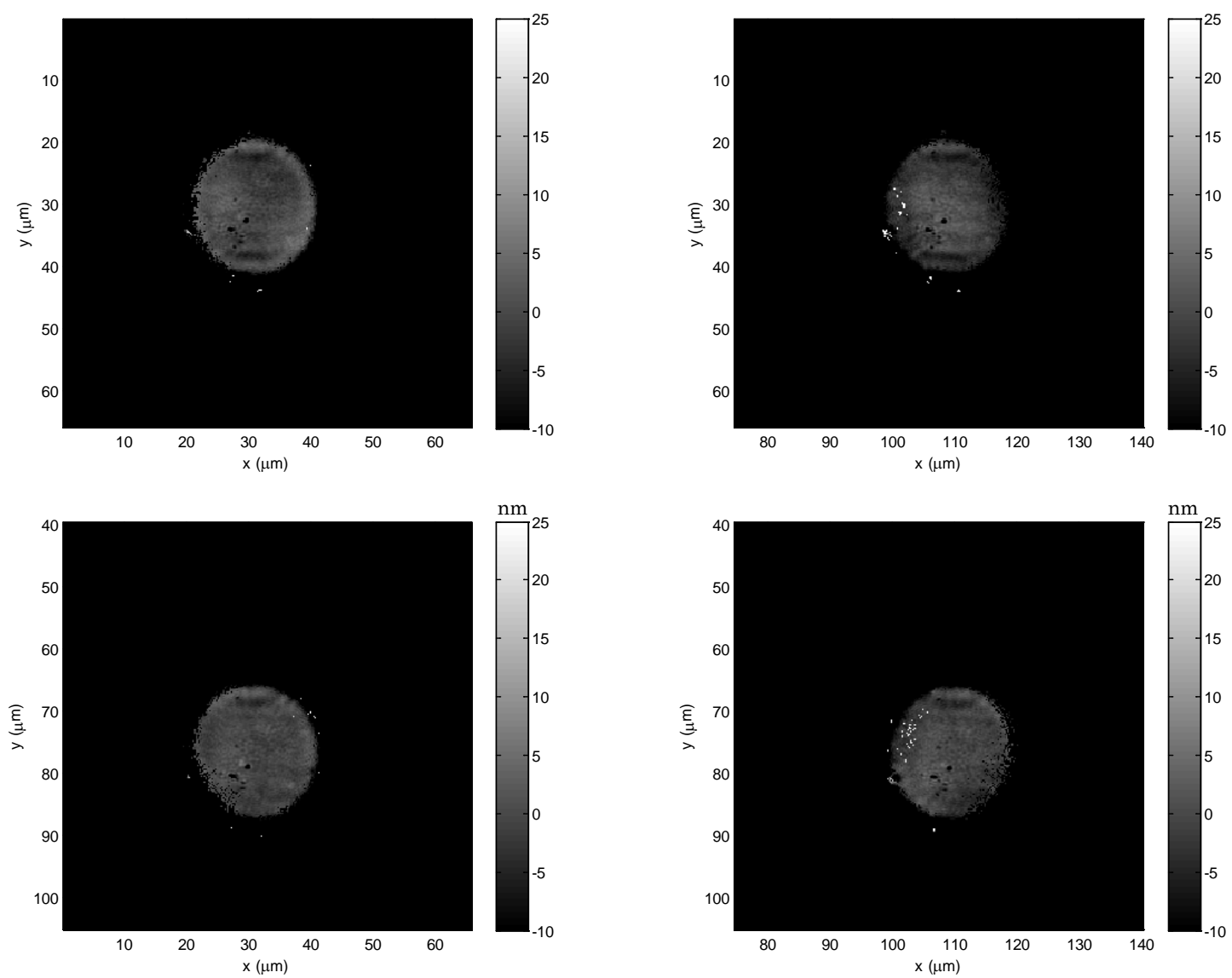

Figure 14: Error surfaces using modified inverse filter at positions CBDE (clockwise from top left) 